\title{
Digitalisierung verändert die Welt der CFOs
}

\author{
Finanzvorstände sehen sie als Effizienztreiber und eine große Chance. Vor allem die Organisations- \\ struktur der Unternehmen dürfte sich entscheidend weiterentwickeln.
}

Die Digitalisierung hat wesentliche Auswirkung auf die Finanzprozesse sowie die Organisationsstruktur ihrer Unternehmen. Davon sind 75 Prozent der Teilnehmer einer Studie der Management- und Technologieberatung Bearing Point überzeugt. Für die Studie wurden 65 CFOs aus unterschiedlichen Industriezweigen Europas befragt. Die Finanzvorstände verstehen demnach die Digitalisierung vor allem als Chance und Hilfe für ihren Bereich. Denn dieser muss aus dem in allen Organisationen zunehmenden „Datendschungel“ und den riesigen Datenmengen aussagekräftige Informationen als Grundlage für Entscheidungen liefern - und das bei gleichbleibenden oder gar sinkenden Kosten.

Verbesserungspotenzial sehen die befragten CFOs derzeit vor allem noch in den Bereichen Datenanalyse (78 Prozent),
Datensicherheit (56 Prozent) und anwenderorientierte Darstellung von Informationen (54 Prozent). Dem Einsatzbereich der sogenannten Collaboration Tools wird ebenfalls erhebliches Potenzial beigemessen. Diese Tools ermöglichen dem CFO, trotz vermehrter Zentralisierung von Aufgaben wie z. B. in Shared Services den Kontakt zum Geschäft nicht zu verlieren. Hierfür werden digitale Werkzeuge benötigt.

Laut Bearing Point sollten sich Finanzvorstände den neuen Möglichkeiten von den spezifischen Anforderungen des Unternehmens und der bestehenden Strukturen her nähern und die Veränderungen dann eher aufgaben- als technologiegetrieben angehen.

Mehr dazu finden Sie auf:

SfP $^{*}$ www.springerprofessional.de/4917038

\section{Springer Gabler}

\section{Empfohlen vom BVBC - Bundesverband der Bilanzbuchhalter und Controller e.V.}

2011, 1235S. Geb.

$€(D) 149,95|€(A) 154,15|{ }^{*} \mathrm{~s} F r \quad 187,00$

ISBN 978-3-8349-1989-2

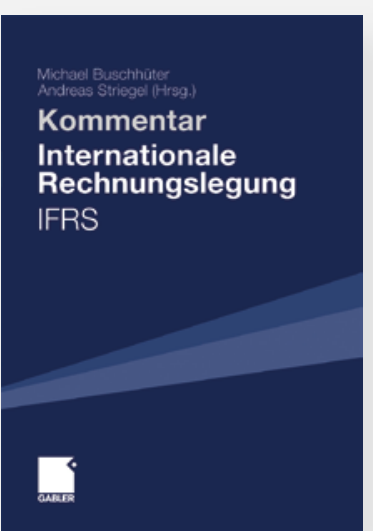

Michael Buschhüter, Andreas Striegel (Hrsg.) Internationale Rechnungslegung - IFRS Kommentar In Deutschland sind alle kapitalmarktorientierten Unternehmen zur Aufstellung von IFRS Abschlüssen verpflichtet. Zahlreiche weitere Unternehmen stellen IFRS Abschlüsse auf freiwilliger Basis auf. Unternehmen und deren Berater müssen daher die IFRS sicher beherrschen. Dieser Kommentar ist ein wichtiger Ratgeber in allen Fragen der Praxis. 